\title{
Review on oral methotrexate in medical management of ectopic pregnancy: a 2 years experience in hospital Shah Alam
}

\section{Wan Nurul Ezyani Wan Jabarudin*, Normala Mohammad Som, Afidatul Mardhiah Mohamad Daud, Nor Dalila Shamsuddin, Nazian Hanna Yaacob, Hamidah Omar}

Department of Obstetrics and Gynecology, Hospital Shah Alam, Selangor, Malaysia

Received: 30 May 2019

Accepted: 02 July 2019

\section{*Correspondence:}

Dr. Wan Nurul Ezyani Wan Jabarudin,

E-mail: ezyani@gmail.com

Copyright: () the author(s), publisher and licensee Medip Academy. This is an open-access article distributed under the terms of the Creative Commons Attribution Non-Commercial License, which permits unrestricted non-commercial use, distribution, and reproduction in any medium, provided the original work is properly cited.

\section{ABSTRACT}

Background: The objective of this study is to determine the success rate of oral methotrexate in Hospital Shah Alam for the past 2 years.

Methods: This is a cross-sectional study using secondary data obtained from medical record office in Hospital Shah Alam on patients with ectopic pregnancy. A total of 35 patients who fulfilled the criteria for medical management were selected. They were prescribed with oral methotrexate with the dose of $60 \mathrm{mg}$ given in 3 divided doses every 2 hours using the standard tablet of $2.5 \mathrm{mg}$. Follow up was done at day 4 , day 7 and till HCG level achieve less than 20 iu/litre.

Results: 29 patients were successfully treated with oral methotrexate $60 \mathrm{mg}(82.8 \%)$. Another 6 patients had to undergo laparoscopic surgery with confirmed leaking, ruptured tubal pregnancy. 4 patients needed second dose of Methotrexate due to rising HCG level and all of them were successfully treated after the second dose. The side effects of oral methotrexate were tolerated well by all patients. There are numbers of predictors for success which are the level of HCG $<4000 \mathrm{iu} / \mathrm{litre}$, size of mass $<4 \mathrm{~cm}$, no abdominal pain during early presentation and decrease of HCG level in between day 4 to day 7 after oral methotrexate.

Conclusions: The success rate of oral methotrexate for stable ectopic pregnancy is good making it a suitable option for clinical settings which has no specialised equipment to handle cytotoxic drugs.

Keywords: Ectopic pregnancy, Conservative management in ectopic pregnancy, Oral methotrexate, Stable ectopic pregnancy, Single dose methotrexate, Tubal pregnancy

\section{INTRODUCTION}

An ectopic pregnancy is any pregnancy implanted outside of the endometrial cavity. In the UK, the incidence is approximately $11 / 1000$ pregnancies, with an estimated 11 000 ectopic pregnancies diagnosed each year. Risk factors for ectopic pregnancy include previous ectopic pregnancy, tubal damage following surgery or infection, smoking and pregnancy conceived via assisted reproductive technique. However, most women with an ectopic pregnancy has no identifiable risk factor. ${ }^{1}$ Any patients who came in early pregnancy with per vaginal bleeding and pain should be investigated for ectopic pregnancy.

Diagnosis of ectopic pregnancy can be facilitated with ultrasound and conjunction of HCG level. Transvaginal ultrasound allows clear visualization of normal or abnormal pregnancy at early stage of pregnancy. ${ }^{2}$ 
Presence of extra uterine sac with life embryo is diagnostic of ectopic. Another finding through transvaginal ultrasound is visualization of adnexa mass suggestive of ectopic pregnancy with predictive value of $63 \%$ up to $100 \% .^{3}$ The other features suggestive of ectopic pregnancy are empty uterus or pseudo sac at level of HCG greater than $1500 \mathrm{IU} / \mathrm{L}$ or presence of free fluid in the absence of intrauterine pregnancy. ${ }^{4}$

Serum HCG can be detected as early as 1 week before expected menstrual period. In a viable intrauterine pregnancy, HCG rapidly increase every 2 days. Cut-off point can be taken as more than $66 \%$ increment over 24 hours for viability. For non-viable intrauterine pregnancy or ectopic pregnancy, HCG will have suboptimal rise. However, $10-15 \%$ viable intrauterine pregnancy might show suboptimal rise. Hence the diagnosis must be correlated with clinical, ultrasound findings and $\mathrm{HCG}$ level. ${ }^{4}$

Ectopic pregnancy can be managed by surgical or nonsurgical (expectant or medical management). Expectant management is based on assumption that the ectopic pregnancy is failing and can regress without any treatment. This option is suitable for stable and asymptomatic patient. The criteria for expectant management are, $\mathrm{HCG}$ at the time of presentation $<1000 \mathrm{IU} / \mathrm{L}$, adnexal mass less than $4 \mathrm{~cm}$ on TVS, and availability of a dedicated unit for serial outpatient follow up with TVS and HCG monitoring.

Medical management is an option of treatment in selected patient. Methotrexate is the medication used in medical management. It can be administered via parenteral or oral. It can be given either via single dose regime or multiple fixed dose regime. Medical management can only be offered in facilities with regular outpatient visits and patient's willingness to come for serial follow up. ${ }^{5}$

NICE guideline recommends that methotrexate should be the first-line management for women who are able to return for follow-up, no significant pain, an unruptured ectopic pregnancy, mass smaller than $35 \mathrm{~mm}$ with no visible heartbeat, a serum HCG between 1500 and 5000 $\mathrm{iu} / \mathrm{l}$ and no intrauterine pregnancy(as confirmed on ultrasound scan). ${ }^{6,7}$

Risk of failure for systemic methotrexate treatment will be increase if the patient has a high initial HCG level of more than $5000 \mathrm{iu} / \mathrm{L}$, an ultrasound pelvis showing a moderate to large free fluid, presence of fetal cardiac activity, and an increase in serum HCG over a 48 hour period. $^{8}$

Surgical management offers significant advantage on management of ectopic pregnancy compared to nonsurgical management. The advantages are that firm diagnosis of ectopic can be made, followed by intervention. The follow up management is shorter in duration and less demanding. Another significant advantage is that, patient can attempt to conceive as soon as they recover from surgery, while patient has to wait for 3 months after the medical treatment in view of potential teratogenicity of methotrexate. ${ }^{9}$

Treatment must be tailored to each patient based on their clinical condition and patient's decision after being counselled regarding risks and benefits of each management option either surgical or medical management. As a new secunder hospital, MTX is only available in oral form in Hospital Shah Alam. Therefore, this study was conducted to determine the success rate of oral MTX in the treatment of ectopic pregnancy in Hospital Shah Alam, Selangor.

\section{METHODS}

A cross sectional study on cases of ectopic pregnancy and pregnancy of unknown location that has been managed using oral methotrexate done by Obstetrics \& Gynaecology Department in Hospital Shah Alam in period of 2 years was conducted from June 2016 till June 2018. A secondary data was retrieved from Medical Record Department Hospital Shah Alam using a standardized data collection form.

The inclusion criteria for medical management included haemodynamically stable ectopic pregnancies with HCG level less than $5000 \mathrm{iu} / \mathrm{L}$, no fetal cardiac activity seen, a mass smaller than $40 \mathrm{~mm}$, normal baseline liver function test, no significant pain, no known sensitivity to MTX and willingness to attend long outpatient follow up. Patients with ruptured ectopic, HCG of more than 5000 $\mathrm{iu} / \mathrm{L}$, a mass more that $40 \mathrm{~mm}$, a presence of heartbeat and abnormal liver function test are excluded from receiving medical treatment and will be subjected for surgical management.

All these patients were administered a fixed dose of oral methotrexate $60 \mathrm{mg} /$ day given in divided dose of $20 \mathrm{mg}$ every 2-hour using standard $2.5 \mathrm{mg}$ tablet. The level of HCG level was monitored day 4, day then weekly post treatment till HCG level become less than $25 \mathrm{iu} / \mathrm{L}$. Those patients with increment of HCG level will be subjected for second dose of oral methotrexate provided they are still fulfilling the criteria for medical management.Information on the side effects and the number of patients who failed or succeeded the medical treatment were obtained from these secondary data.

\section{Statistical analysis}

All of these data were then analysed using using descriptive statistics via IBM SPSS version 20.

\section{RESULTS}

The total number of ectopic cases encountered from June 2016 to June 2018 is 338 cases with total deliveries of 20690 deliveries. 
Table 1: The percentages of successful cases in the study.

\begin{tabular}{|lll|}
\hline Successful & Yes & No \\
\hline No. of patient & $29(82.8 \%)$ & $6(17.8 \%)$ \\
\hline
\end{tabular}

The number of patients included in this case series was 35 patients. Table 1 showed that 29 patients $(82.8 \%)$ were successfully treated where the level of HCG decreased more than $15 \%$ of pre-treatment HCG level. There were 4 patients who required a second cycle of methotrexate in view of increasing HCG level and all of them were successfully treated with methotrexate. Another 6 patients (17.8\%) needed emergency surgery for unstable ectopic pregnancy.

Table 2: Ultrasonography site of ectopic pregnancy.

\begin{tabular}{|ll|}
\hline Site of ectopic pregnancy & Result \\
\hline Scar pregnancy & 1 \\
\hline Cervical pregnancy & 1 \\
\hline Cornual pregnancy & 1 \\
\hline Pregnancy of unknown location & 7 \\
\hline Adnexal pregnancy & 25 \\
\hline
\end{tabular}

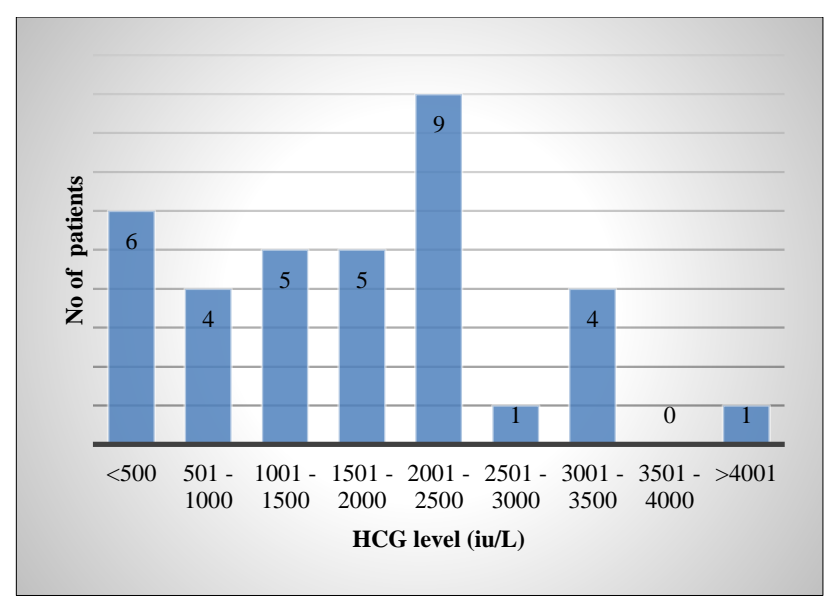

Figure 1: Level of HCG pre-treatment.

Table 2 showed the ultrasonography site of the ectopic pregnancies in this study. As expected, 25 patients (71.4\%) were diagnosed via ultrasound with adnexal pregnancy. 7 patients $(20 \%)$ were diagnosed with pregnancy of unknown location. We have also successfully treated a scar pregnancy (2\%), a cornual pregnancy $(2 \%)$ and a cervical pregnancy $(2 \%)$ that had fulfilled the inclusion criteria.

Table 3: BMI level of patients in these studies.

\begin{tabular}{|llllll|l|}
\hline BMI & Underweight & Normal & Overweight & Obese I & Obese II & Obese III \\
\hline & $<18.5$ & $18.5-24.9$ & $25.0-29.9$ & $30.0-34.9$ & $35.0-39.9$ & $>40.0$ \\
\hline Result & 2 & 13 & 9 & 7 & 4 & \\
\hline
\end{tabular}

The total HCG level at the start of treatment ranges from $126 \mathrm{iu} / \mathrm{L}$ up till $5279 \mathrm{iu} / \mathrm{L}$ as shown in Figure 1. 17 patients' HCG level (48\%) decreased more than $15 \%$ of the initial HCG level after 7 days of initiating treatment. The mean of initial HCG level for both the success group and the fail group was $1682 \mathrm{iu} / \mathrm{L}$ and $2438 \mathrm{iu} / \mathrm{L}$ respectively with p-value of 0.295 .

Table 4: The presence of free fluid during ultrasound examination between the success and fail group.

\begin{tabular}{|lll|}
\hline Presence of free fluid & Success & Fail \\
\hline No & 22 & 1 \\
\hline Yes & 7 & 5 \\
\hline
\end{tabular}

Table 3 showed the BMI level of patients in this study. As mentioned previously, treatment regime consisted of a fixed single dose of $60 \mathrm{mg} /$ day given in 3 divided doses over 2-hour intervals, the objective was to demonstrate if there is any significant difference between the success group and the failure group by using Chi-square test in relation to $\mathrm{BMI}$. The p-value demonstrated is 0.788 , hence proving that BMI is not significant to the success rate of medical management in ectopic pregnancy.

Table 4 shows the number of patients with presence of free fluid seen during ultrasound exam tabulated between the success and failure group. The presence of free fluid has been found to be a good indicator for predictor of success as patients that presented with free fluid during ultrasound examination are more likely to fail. The pvalue using Chi- square test was 0.005 .

There were no documented side effects expressed by the patients during treatments and follow-up throughout these 2 years.

\section{DISCUSSION}

Methotrexate is a chemotherapeutic agent by acting as an anti-neoplastic anti metabolite with immunosuppressant properties10. Its usage in gynaecology was first reported by $\mathrm{Li}$ and Heartz in 1956 where it was used to cure women with terminal choriocarcinoma. ${ }^{11}$ 
Mechanism of action of methotrexate is by blocking the DNA synthesis as it works as a folate antagonist by blocking the folic acid binding site on the enzyme dihydrofolate reductase thus rendering it inactive hence conversion of folic acid to tetrahydrofolic acid cannot occur. Tetrahydrofolic acid is crucial in the DNA synthesis process as it contribute the one-carbon transfer reaction needed by the coenzymes. ${ }^{5}$

The first documented usage of methotrexate in treating ectopic pregnancy was in 1982. The dosage used then was modified from the treatment of choriocarcinoma. It was given using multiple dose protocol where patient was administered an intramuscular injection of $1 \mathrm{mg} / \mathrm{kg}$ of methotrexate with $0.1 \mathrm{mg} / \mathrm{kg}$ leucovorin factor rescue 24 hours later. ${ }^{12}$

There are few recommendations regarding the best dose regimes for methotrexate in the management of ectopic pregnancy either single dose protocol or multiple dose protocol. ${ }^{13}$ The dosage used in the single dose regime are higher while multiple fixed dose regime will used lower dosage of medication over a period of time. The overall success rate of intramuscular multi-dose treatment in a meta-analysis by Barnhart et al is reported as $92.7 \%$ (241 of 260 patients) with a $95 \%$ CI $89-96 \% .^{14}$

In view of risk of side effects, the single-dose protocol was proposed in 1991 consist of $50 \mathrm{mg} / \mathrm{m}^{2}$ of methotrexate given intramuscularly, administered on day 0 . There were thirty patients enrolled in this study and 29 patients, or $96.7 \%$ were successfully treated. ${ }^{15}$ In 1993 the study was expanded to 120 patients whom all were administered single dose methotrexate treatment with good results. 113 patients out of 120 were successfully treated with single dose of methotrexate while the rest required a second dose of methotrexate on day $7 .{ }^{16}$

There are various dosages that has been used for oral methotrexate regimen varies from lowest dose of $2.5 \mathrm{mg}$ daily which is not more effective than placebo up to 20 $\mathrm{mg}$ daily for 5 days (for multiple fixed regimen) which is more successful. ${ }^{17}$ The most common regime used for oral single fixed dose regime would be $60 \mathrm{mg} / \mathrm{m} 2$ with success rate of $86 \% .^{18}$

In view of no final consensus mentioning the most recommended dosage for oral methotrexate, $60 \mathrm{mg} /$ day had been used in our institution in divided dose using a standard $2.5 \mathrm{mg}$ tablet [ 8 tablet $(20 \mathrm{mg})$ every two hours $x$ 3] instead of using the most recommended dose of $60 \mathrm{mg} / \mathrm{m}^{2}$ for single fixed dose for oral methotrexate.

The success rate in this study of $82.8 \%$ is slightly lower than the success rate of previous reported study of $86 \%$ whereby they used oral methotrexate $60 \mathrm{mg} / \mathrm{m}^{2} .^{18}$

The 6 patients who failed the treatment, 5 of them had complaint of lower abdominal pain and has presence of free fluid during USG prior to treatment, one patient had mass of more than $4 \mathrm{~cm}$, while another one has a high HCG level of $5086 \mathrm{iu} / \mathrm{L}$ before taking the methotrexate but they were treated with medical management in view of previous history of ectopic pregnancy treated surgically. This was an attempt to try to preserve their fertility.

According to this study, the BMI of the patient is not a significant factor in the treatment as there was no significant difference of the BMI between the success group and the failure group.

The main predictor factors to determine the success rate of medical management would be the level of HCG less than $4000 \mathrm{iu} / \mathrm{L}$, size mass of $4 \mathrm{~cm}$, no free fluid during ultrasound scan, and no abdominal pain during early presentation and decrement of BHCG level between day 4 to day 7 post treatment. ${ }^{19,20}$

Systemic methotrexate is also a good option for stable non tubal ectopic pregnancy such as cervical, cornual and scar ectopic. This is provided that these patients fulfilled the inclusion criteria and factors that determining the success rate of medical management. ${ }^{21,22}$

The advantages of using lower dose of methotrexate are the reduction in side effect of methotrexate and folic acid rescue was not needed. ${ }^{5}$ The advantage of oral methotrexate over intramuscular methotrexate is that the administration via intramuscular route causes pain and local reaction to surrounding soft tissues. Oral methotrexate is more cost effective as we need less equipment and expertise (pharmacist in handling parenteral cytotoxic drug). The preparation and the disposition require the personnel to wear PPE and special fume hood to prepare the drug.

In this study, there was no hysterosalpingogram done to assess tubal patency post treatment. This is an important aspect that needs to be done in the future as it will determine the effectiveness of methotrexate in preserving fertility especially in those who have had previous ectopic. Eventually, out of 30 patients that had been treated, 3 patients conceived a normal intrauterine pregnancy after treatments.

\section{CONCLUSION}

In conclusion, the inaccessibility to parenteral methotrexate has opened a new horizon to explore other option which will benefit more patients in the future. Oral methotrexate can be used successfully to treat ectopic pregnancy and there are few advantages over intramuscular methotrexate.

\section{ACKNOWLEDGMENTS}

Authors would like to thank the Director General of Health Malaysia for the permission to publish this paper. 
Funding: No funding sources

Conflict of interest: None declared

Ethical approval: The study was approved by the Institutional Ethics Committee

\section{REFERENCES}

1. Elson CJ, Salim R, Potdar N, Chetty M, Ross JA, Kirk EJ on behalf of the Royal College of Obstetricians and Gynaecologists. Diagnosis and management of ectopic pregnancy. BJOG. 2016;123(13):e15-e55.

2. Kirk E, Papageorghiou AT, Condous G, Tan L, Bora $\mathrm{S}$, Bourne $\mathrm{T}$. The diagnostic effectiveness of an initial transvaginal scan in detecting ectopic pregnancy. Hum Reprod. 2007;22(11):2824-8.

3. Condous G, Okaro E, Khalid A, Lu C, Van Huffel S, Timmerman D. et al. The accuracy of transvaginal ultrasonography for the diagnosis of ectopic pregnancy prior to surgery. Human Reproduction, 2005;20(5):1404-9.

4. Luesley D, Cardozo BP. Obstetrics and gynaecology: An evidence-based text for MRCOG (3rd Edition). London: Hodder Arnold. 2016;685-698.

5. Murray H. Diagnosis and Treatment of Ectopic Pregnancy. Canadian Med Asso J. 2005;173(8):90512.

6. National Collaborating Centre for Women's and Children's Health (UK). Ectopic Pregnancy and Miscarriage: Diagnosis and Initial Management in Early Pregnancy of Ectopic Pregnancy and Miscarriage. London: Royal College of Obstetrics and Gynecology; 2019 April (NICE Clinical Guidelines, No. 126). Available at: https://www.nice.org.uk/guidance/ng126/resources/e ctopic-pregnancy-and-miscarriage-diagnosis-andinitial-management-pdf-66141662244037. Accessed on 7th May 2019.

7. Megan B. Potter, Lisa A. Lepine, Denise J. Jamieson. Predictors of success with methotrexate treatment of tubal ectopic pregnancy at Grady Memorial Hospital. Am J Obstet Gynecol. 2003:188(5):1192-4.

8. Menon S, Colins J, Barnhart KT. Establishing a human chorionic gonadotropin cutoff to guide methotrexate treatment of ectopic pregnancy: a systematic review. Fertil Steril. 2007;87(3):481-4.

9. Sowter MC, Farquhar CM, Petrie KJ, Gudex G. A randomised trial comparing single dose systemic methotrexate and laparoscopic surgery for the treatment of unruptured tubal pregnancy. BJOG. 2001;108(2):192-203.

10. Stika, Catherine S. Methotrexate: the pharmacology behind medical treatment for ectopic pregnancy. Clin Obstet Gynecol. 2012;55(2):433-9.
11. Li MC, Hertz R, Bergenstal DM. Therapy of Choriocarcinoma and related trophoblastic tumors with folic acid and purine antagonists. New England J Med. 1958;259(2):66-74.

12. Tanaka T, Hayashi H, Kutsuzawa T. Treatment of interstitial ectopic pregnancy with methotrexate: report of a successful case. Fertil Steril. 1982;37(6):851-2.

13. Bachman EA, Barnhart K. Medical management of ectopic pregnancy. Clin Obstet Gynecol. 2012;55(2):440-7.

14. Barnhart KT, Gosman G, Ashby R, Sammel M. The medical management of ectopic pregnancy: a metaanalysis comparing "single dose" and "multidose" regimens. Obstet Gynecol. 2003;101(4):778-84.

15. Stovall TG, Ling FW, Gray LA Single-dose methotrexate for treatment of ectopic pregnancy. Obstet Gynecol. 1991;77(5):754-7.

16. Stovall TG, Ling FW Single-dose methotrexate: an expanded clinical trial. Am J Obstet Gynecol. 1993;168(6 Pt 1):1759-62.

17. Korhonen J, Stenman U, Ylostalo P. Low-dose oral methotrexate with expectant management of ectopic pregnancy. Obstet Gynecol. 1996;88(5):775-8.

18. Lipscomb G, Meyer NL, Flynn DE, Peterson M, Ling F. Oral methotrexate for treatment of ectopic pregnancy. Am J Obstet Gynecol. 2002;186(6):11925.

19. Trio D, Strobelt N, Picciolo C, Lapinski RH, Ghidini A. Prognostic factors for successful expectant management of ectopic pregnancy. Fertil Steril. 1995;63(3):469-72.

20. Skubisz MM, Li J, Wallace EM, Tong S. Decline in hCG levels between days 0 and 4 after a single dose of methotrexate for ectopic pregnancy predicts treatment success: a retrospective cohort study. BJOG. 2011;118(13):1665-8.

21. Jurkovic D, Hillaby K, Woelfer B, Lawrence A, Salim R, Elson CJ. First-trimester diagnosis and management of pregnancies implanted into the lower uterine segment Cesarean section scar. Ultrasound Obstet Gynecol. 2003;21(3):220-7.

22. Peng P, Gui T, Liu X, Chen W, Liu Z. Comparative efficacy and safety of local and systemic methotrexate injection in cesarean scar pregnancy. Ther Clin Risk Manag. 2015;11:137-42.

Cite this article as: Jabarudin WNEW, Som NM, Daud AMM, Shamsuddin ND, Yaacob NHY, Omar $\mathrm{H}$. Review on oral methotrexate in medical management of ectopic pregnancy: a 2 years experience in hospital Shah Alam. Int J Reprod Contracept Obstet Gynecol 2019;8:3229-33. 